\title{
Microbial Signature in Adipose Tissue of Crohn's Disease Patients
}

\author{
Carolina Serena ${ }^{1,2,+} \mathbb{D}$, Maribel Queipo-Ortuño ${ }^{3,4,+} \mathbb{D}$, Monica Millan ${ }^{5,6}$, \\ Lidia Sanchez-Alcoholado ${ }^{3,4}$, Aleidis Caro ${ }^{5}$, , Beatriz Espina ${ }^{5}$, Margarita Menacho ${ }^{7}$, \\ Michelle Bautista ${ }^{7}$, Diandra Monfort-Ferré ${ }^{1}$, Margarida Terrón-Puig ${ }^{1,2}$, Catalina Núñez-Roa ${ }^{1,2}$, \\ Elsa Maymó-Masip ${ }^{1,2}$, M. Mar Rodriguez ${ }^{1,2}$, Francisco J. Tinahones ${ }^{8}$, Eloy Espin ${ }^{9}$, \\ Marc Martí ${ }^{9}$, Sonia Fernández-Veledo ${ }^{1,2, *}$ and Joan Vendrell $1,2,10, *$
}

1 Hospital Universitari de Tarragona Joan XXIII, Institut d'Investigació Sanitària Pere Virgili Universitat Rovira i Virgili, 43005 Tarragona, Spain; carolina.serena@iispv.cat (C.S.); diandramonfort@gmail.com (D.M.-F.); margarida.terron@gmail.com (M.T.-P.); catinuroa@gmail.com (C.N.-R.); elsamaymomasip@gmail.com (E.M.-M.); m.marrodpe@gmail.com (M.M.R.)

2 CIBER de Diabetes y Enfermedades Metabólicas Asociadas (CIBERDEM), Instituto de Salud Carlos III, 08029 Madrid, Spain

3 CIBER de Obesidad y Nutrición (CIBERObN), Instituto de Salud Carlos III, 08029 Madrid, Spain; maribelqo@gmail.com (M.Q.-O.); 1.s.alcoholado@gmail.com (L.S.-A.)

4 Unidad de Gestión Clínica Intercentros de Oncología Medica, Hospitales Universitarios Regional y Virgen de la Victoria de Málaga, Instituto de Investigación Biomédica de Málaga (IBIMA)-CIMES-UMA, 29010 Málaga, Spain

5 Colorectal Surgery Unit, Hospital Universitari Joan XXIII, 43007 Tarragona, Spain; monica.millan@ymail.com (M.M.); dra5028@gmail.com (A.C.); bespina84@gmail.com (B.E.)

6 Colorectal Surgery Unit, Hospital Universitari La Fe, 46026 Valencia, Spain

7 Digestive Unit, University Hospital Joan XXIII, IISPV, 43007 Tarragona, Spain; mmenacho.hj23.ics@gencat.cat (M.M.); bmichelleannette@gmail.com (M.B.)

8 Endocrinology and Nutrition Department, Biomedical Research Institute from Malaga (IBIMA), University Hospital Virgen de la Victoria of Malaga, Malaga University, 29010 Málaga, Spain; fjtinahones@uma.es

9 Colorectal Surgery Unit, General Surgery Service, Valle Hebron Hospital, Autonomous University of Barcelona, 08035 Barcelona, Spain; eespin@vhebron.net (E.E.); marcmartig@gmail.com (M.M.)

10 Medicine and Surgery Department, School of Medicine, Universitat Rovira i Virgili, 43005 Tarragona, Spain

* Correspondence: sonia.fernandezveledo@gmail.com (S.F.-V.); jvo@comt.es (J.V.); Tel.: +34-977-29-58-00 (ext. 3401) (S.F.-V. \& J.V.); Fax: +34-977-29-58-23 (S.F.-V. \& J.V.)

+ Co-first authors.

Received: 11 May 2020; Accepted: 29 July 2020; Published: 31 July 2020

\begin{abstract}
Crohn's disease (CD) is characterized by compromised immune tolerance to the intestinal commensal microbiota, intestinal barrier inflammation, and hyperplasia of creeping fat (CF) and mesenteric adipose tissue (AT), which seems to be directly related to disease activity. Gut microbiota dysbiosis might be a determining factor in CD etiology, manifesting as a low microbial diversity and a high abundance of potentially pathogenic bacteria. We tested the hypothesis that CF is a reservoir of bacteria through 16S-rRNA sequencing of several AT depots of patients with active and inactive disease and controls. We found a microbiome signature within $\mathrm{CF}$ and mesenteric AT from patients, but not in subcutaneous fat. We failed to detect bacterial DNA in any fat depot of controls. Proteobacteria was the most abundant phylum in both CF and mesenteric AT, and positively correlated with fecal calprotectin/C-reactive protein. Notably, the clinical status of patients seemed to be related to the microbiome signature, as those with the inactive disease showed a reduction in the abundance of pathogenic bacteria. Predictive functional profiling revealed many metabolic pathways including lipopolysaccharide biosynthesis and sulfur metabolism overrepresented in active $\mathrm{CD}$ relative to that in inactive $\mathrm{CD}$. Our findings demonstrate that microbiota dysbiosis associated with $\mathrm{CD}$ pathophysiology is reflected in AT and might contribute to disease severity.
\end{abstract}


Keywords: tissue microbiota; inflammatory bowel disease; $16 \mathrm{~S}$ sequencing; creeping fat; PICRUSt analysis; Escherichia coli; Fusobacterium; lipopolysaccharide biosynthesis

\section{Introduction}

Inflammatory bowel diseases (IBDs) have become a worldwide epidemic in developed societies. They are a heterogeneous group of disorders of multifactorial etiology and are commonly represented by two major phenotypes-Crohn's disease (CD) and ulcerative colitis (UC) — both characterized by persistent inflammation and ulcerations at the small or large bowel, a low rate of spontaneous remission, and an undulating course of activity with relapsing attacks after periods of remission. Dysbiosis and decreased complexity of the intestinal microbiota, owing to a shift in the balance between commensal and potentially pathogenic microorganisms, are well-recognized features in both CD and UC [1-5], with microbiota dysbiosis being significantly greater in CD than in UC [6]. Indeed, the gut microbiota is believed to be a driving factor for inflammation and the development of intestinal lesions, because the surgical diversion of the fecal stream has been shown to induce remission [7-10]. Mesenteric and intestinal disease manifestations are tightly coupled in CD and, in this context, some authors have recently claimed that the mesentery is a contiguous organ that may play an important role in immunological processes due to its anatomical position in the intestinal tract [11-14]. Hypertrophy of the mesenteric fat adjacent to the inflamed regions of the intestine, so-called "creeping fat (CF)" is a hallmark of CD and seems to be directly related to disease activity $[15,16]$. Indeed, it has been recently described that reoperation rates among patients with CD decrease dramatically, from $27 \%$ to $2.7 \%$, when the mesentery is included during intestinal resection [13]. Mesenteric adipose tissue (AT) expansion in CD is mainly dependent on adipocyte hyperplasia, which occurs via recruitment and differentiation of AT precursors termed AT-derived stem cells (ASCs) $[17,18]$. ASCs not only participates in the turnover of mature adipocytes in humans, but they also possess immunoregulatory properties that can be induced by the underlying pathological state $[19,20]$. In this context, we recently demonstrated that ASCs isolated from patients with CD have augmented proliferative, invasive, and phagocytic capacities and seem to be key players in the development of CF [21].

Bacterial translocation and tissue microbiota in humans are subjects of intense debate since several years ago [18,22-25]. Although some authors hypothesize that adipose tissue microbiota may come from the intestine when leaky gut occurs, there is still no reliable evidence in $\mathrm{CD}$. Thus, previous works identified the presence of bacterial translocation $[18,26,27]$ instead of determining the adipose tissue microbiota profile, and none describes the evolutive changes according to the clinical status of CD patients.

In this study, we show that mesenteric AT is a bacterial reservoir and that patients with $\mathrm{CD}$ have a microbiome signature within mesenteric AT that is dependent on their clinical status. Accordingly, mesenteric AT might cooperate with the gut in a pro-inflammatory feedback loop, generating a specific microbiome signature in this tissue to influence $\mathrm{CD}$ status and/or disease progression.

\section{Material and Methods}

\subsection{Study Design}

Subjects were recruited at University Hospital Joan XXIII (Tarragona, Spain) and University Hospital Vall d'Hebrón (Barcelona, Spain) following the tenets of the Declaration of Helsinki. The corresponding hospital ethics committees approved the study, and written informed consent was obtained from all participants before entering the study. Donors were classified as those in relapse (active) or remission (inactive) following the Crohn's Disease Activity Index criteria (CDAI score) as well as biological and clinical parameters such as PCR and fecal calprotectin. Furthermore, endoscopic 
evaluation was available in $75 \%$ of the patients, with a complete correspondence with the clinical classification obtained by CDAI [28,29].

Samples of mesenteric (MES-VAT) and subcutaneous (SAT) AT were obtained from patients with inactive $C D$ undergoing non-acute surgical interventions such as hernia repair or cholecystectomy, in a scheduled routine surgery $(n=5)$. Moreover, samples of mesenteric pericolic "wrapping" AT (CF origin; CF-VAT), MES-VAT (away from the active lesion in a healthy mesentery), and SAT $(n=8)$ were obtained from patients with active or complicated CD submitted to surgery. Lastly, samples of $M E S$-VAT and SAT were obtained from non-CD subjects (control group) undergoing non-acute surgical interventions such as hernia repair or cholecystectomy, in a scheduled routine surgery $(n=8)$. Tissue samples were aseptically collected and (when appropriate) all were obtained before bowel opening or resection to avoid possible contamination. Clinical data, anthropometric, and biochemical variables from the cohort are presented in Table 1.

Table 1. Demographic and clinical characteristics of the cohort.

\begin{tabular}{|c|c|c|c|}
\hline & Control & Inactive CD & Active CD \\
\hline$n$ & 8 & 5 & 8 \\
\hline Sex (male/female) & $4 / 4$ & $2 / 3$ & $4 / 4$ \\
\hline Age (years) & $46.1(37.2-55.1)$ & $47.6(35.2-60.1)$ & $45.8(35.4-58.5)$ \\
\hline BMI $\left(\mathrm{kg} / \mathrm{m}^{2}\right)$ & $25.8(20.2-29.1)$ & $21.59(20.1-24.1)$ & $23.8(20.5-27.3)$ \\
\hline Glucose $(\mathrm{mg} / \mathrm{dL})$ & $96.6 \pm 6.3$ & $94.6 \pm 5.4$ & $84.83 \pm 4.7^{\mathrm{a}}$ \\
\hline Smoking, $n(\%)$ & $4(50)$ & $3(60)$ & $4(50)$ \\
\hline Cholesterol (mg/dL) & $131 \pm 11.2$ & $118.2 \pm 10.9$ & $119.2 \pm 15$ \\
\hline HDL (mg/dL) & $30.7 \pm 4.5$ & $31.13 \pm 3.2$ & $31.4 \pm 6.4$ \\
\hline Triglycerides (mg/dL) & $186(167.2-205.5)$ & $186(170.3-199.2)$ & $119.6(100.9-140)^{a, b}$ \\
\hline Insulin $(\mu \mathrm{IU} / \mathrm{mL})$ & $2.05 \pm 1.9$ & $4.98 \pm 1.1^{\mathrm{a}}$ & $6.12 \pm 2.3^{a}$ \\
\hline HOMA-IR & $0.7 \pm 0.4$ & $1.18 \pm 0.5$ & $1.44 \pm 0.7^{\mathrm{a}, \mathrm{b}}$ \\
\hline Age at diagnosis (years) & & $26.5 \pm 6.6$ & $28.1 \pm 8.5$ \\
\hline Time in remission (months) & & $29 \pm 9.3$ & \\
\hline Indication of surgery & $\mathrm{CoH}$ & $\mathrm{CoH}$ & $5 \mathrm{SCD} / 5 \mathrm{FCD}$ \\
\hline Immunomodulator use & & $5 / 5$ & $10 / 10$ \\
\hline Biological agent treatment & & $2 / 5$ & $4 / 10$ \\
\hline Steroid treatment & & $4 / 5$ & $7 / 10$ \\
\hline C-reactive protein $(\mathrm{mg} / \mathrm{dL})$ & $0.05 \pm 0.04$ & $0.3 \pm 0.13^{\mathrm{a}}$ & $3.4 \pm 1.6^{\mathrm{a}, \mathrm{b}}$ \\
\hline Fecal calprotectin $(\mu \mathrm{g} / \mathrm{g})$ & & $81.25 \pm 20.3$ & $2132.4 \pm 159.1^{b}$ \\
\hline
\end{tabular}

Abbreviations: BMI, body mass index; HDLc, high-density lipoprotein cholesterol; $\mathrm{CoH}$, cholecystectomy or hernia; CD; Crohn's disease; SCD, stenotic Crohn's disease; FCD, fistulizing Crohn's disease; HOMA-IR, Homeostatic Model Assessment for Insulin Resistance. Results are presented as mean \pm SD or median (25th-75th percentiles), as appropriate. Differences were analyzed as described in Material and Methods. ${ }^{a}, p<0.01$ versus control group (non-CD subjects). ${ }^{b}, p<0.01$ versus inactive CD subjects.

\subsection{RNA Extraction from Tissues}

RNA was extracted from 200 mg of tissue using the TriPure Isolation Reagent (Roche, Basel, Switzerland). RNA concentration was determined by absorbance at $260 \mathrm{~nm}$, and purity was estimated with a Nanodrop spectrophotometer (Nanodrop Technologies Inc., Wilmington, DE, UAS). cDNA was synthesized using SuperScript II reverse transcriptase and random hexamer primers (Invitrogen Life Technologies, Darmstadt, Germany).

\subsection{PCR Amplification and Analysis of 16S-rRNA Sequences}

For microbial community profiling, 16S-rRNA gene sequences were amplified from cDNA using the 16S Metagenomics Kit (Thermo Fisher Scientific, Madrid, Spain), which includes two primer sets of the $16 \mathrm{~S}$ hypervariable regions in bacteria: primer set V2-4-8 and primer set V3-6, 7-9. PCR products were purified using Agencourt AMPure XP DNA purification beads (Beckman Coulter Genomics $\mathrm{GmbH}$, Bernried, Germany) to remove primer dimers. The concentration and the average size of 
each amplicon were determined using the Quant-iT PicoGreen dsDNA Assay Kit (Invitrogen), and the amount of DNA fragments per microliter was calculated. Libraries were prepared using the Ion Plus Fragment Library Kit, and samples were labeled using the Ion Xpress Barcode Adapters 1-16 kit. Library concentrations were determined using the Ion Universal Library Quantification Kit. Emulsion PCR and sequencing of the amplicon libraries were carried out using the Ion Torrent S5 system and the Ion 520/530 Kit-Chef. After sequencing, the individual sequence reads were filtered using the Ion Reporter Software V4.0 to remove low-quality and polyclonal sequences. All Ion platforms and reagents were from Thermo Fischer Scientific.

\subsection{Bioinformatic Analysis}

Datasets were analyzed using Quantitative Insights into Microbial Ecology (QIIME II) 2017.10.0 software (http://qiime.org). Sequencing reads were de-multiplexed and further filtered through the split_library.py script of QIIME II. To guarantee a higher level of accuracy, the reads were excluded from analysis if they had an average quality score $<25$ and if there were ambiguous base calls. Operational taxonomic units (OTUs) were defined by clustering sequences at a similarity of $>97 \%$ and the representative sequences, chosen as the most abundant in each cluster, were submitted to the UCLUST-consensus taxonomy assigner (https://drive5.com/usearch/manual/uclust_algo.html) to obtain the taxonomy assignment and the relative abundance of each OTU, using the Greengenes 16S rRNA gene database (http://greengenes.lbl.gov/cgi-bin/nph-index.cgi). $\alpha$ - and $\beta$-diversity were evaluated through QIIME II as described [30]. Distances between microbial communities from each sample were calculated with the jackknife coefficient; they were represented by the unweighted pair group method with arithmetic mean algorithm (UPGMA) clustering trees describing the dissimilarity between multiple samples generated by QIIME II. 16S gene sequences have been deposited in GenBank under different accession numbers.

We used PICRUSt analysis to predict metagenome function by picking OTUs against the Greengenes database, as previously described [31,32]. This OTU table was used for predicting metagenomes at different KEGG levels. The statistical analysis was performed in the R environment (v3.3.3). $p$-values were corrected for multiple testing using the Benjamini-Hochberg method [31].

\subsection{Statistical Analysis}

Statistical analysis was performed with the Statistical Package for the Social Sciences software, version 15 (SPSS, Chicago, IL, USA). For clinical and anthropometrical variables, normally distributed data were expressed as mean $\pm \mathrm{SD}$, and variables with no Gaussian distribution values were expressed as median (25th-75th quartiles). Student's $t$-test with Bonferroni adjustment was used to compare the mean value of normally distributed continuous variables. For variables that did not have a Gaussian distribution, we used the Kruskal-Wallis test with post hoc Dunn's multiple comparison test. To analyze the differences in nominal variables between groups, we used the $\chi 2$ test. For microbiota data, the statistical analysis was performed in R v3.3.3. Two-way analysis of variance (ANOVA), followed by Bonferroni's test for multiple comparisons, was performed to check for significant differences. Pearson's correlation coefficient with Bonferroni adjustment was used to analyze the relationship between parameters. Visualizations were performed with GraphPad Prime v6 (GraphPad Software Inc., San Diego, CA, USA).

\subsection{Ethics Approval and Consent to Participate}

The study has been carried out in accordance with the guidelines of the Declaration of Helsinki and the corresponding hospital ethics committees approved the study. Written informed consent was obtained from all participants before entering the study. 


\section{Results}

\subsection{Microbiome Signature of Mesenteric Adipose Tissue in Patients with Crohn's Disease}

Adipose tissue from creeping fat from active CD patients, showed a marked inflammatory infiltration with an increased expression of pro-inflammatory genes (Supplementary Figure S1). Sequencing analysis of the 16S-rRNA gene sequences from these patients revealed a rich microbiome profile both in CF-VAT and in MES-VAT. By contrast, 16S-rRNA gene sequencing was negative in SAT depots of patients with CD (active and inactive) and in MES-VAT and SAT of non-CD subjects (controls) with the method employed in this study.

Weighted UniFrac principal coordinates analysis (PCoA), a measure of community composition, showed that the microbiome of CF-VAT and MES-VAT of patients with active CD had a similar pattern of clustering ( $p=0.502$ ), with the principal scores explaining $26 \%, 13 \%$ and $9 \%$ of the total variation (Figure 1A). No significant differences were found in $\alpha$-diversity indices (Observed $(p=0.109)$, Chao1 $(p=0.115), \operatorname{ACE}(p=0.060)$, Shannon $(p=0.155)$, Simpson $(p=0.065)$, InvSimpson $(p=0.071)$, and Fisher $(p=0.193))$ between the CF-VAT and MES-VAT microbiomes in patients with active CD (Figure 1B). However, we found significant differences in the relative abundance of several taxa between the CF-VAT and MES-VAT. Proteobacteria was the most abundant phylum in both CF-VAT and MES-VAT, followed by Bacteroidetes and Firmicutes. At the phylum level, Proteobacteria relative abundance was significantly higher in CF-VAT than in MES-VAT $(70.64 \pm 7.14 \%$ vs. $52.83 \pm 9.25 \%, p=0.00059)$ (Figure 1C). At the family level, the relative abundance of Enterobacteriaceae was significantly higher in CF-VAT than in MES-VAT (53.47 $\pm 7.16 \%$ vs. $40.29 \pm 7.75 \%, p=0.0091$ ) (Figure 1D), whereas the relative abundance of Bacteroidaceae was significantly lower $(10.93 \pm 7.84 \%$ vs. $23.57 \pm 7.03 \%, p=0.0151)$.

At the genus level, Bacteroides relative abundance was significantly lower in CF-VAT than in MES-VAT (15.53 $\pm 8.84 \%$ vs. $31.59 \pm 10.59 \%, p=0.0234)$ (Figure 1E). Specifically, we found a lower relative abundance of the species Bacteroides vulgatus in CF-VAT relative to that in MES-VAT (1.12 \pm $0.95 \%$ vs. $15.85 \pm 6.96 \%, p=0.0155$ ) (Figure $1 \mathrm{E}$ ).

\subsection{The Microbiome Signature in Mesenteric Adipose Tissue in Crohn's Disease Is Related to the Clinical Status}

Next, we compared the microbiome of MES-VAT between patients with active and inactive disease. Weighted UniFrac PCoA showed a different cluster pattern for MES-VAT between active and inactive $C D$. The analysis of similarities with permutations revealed significant differences between active and inactive CD ( $p=0.047)$, as demonstrated by the two principal component scores, which accounted for $32 \%$ and $21 \%$ of the total variation (Figure $2 \mathrm{~A}$ ). However, no significant differences were observed in $\alpha$-diversity indices (Observed $(p=0.883)$, Chao1 $(p=0.194), \operatorname{ACE}(p=0.476)$, Shannon $(p=0.379)$, Simpson $(p=0.103)$, InvSimpson $(p=0.106)$, and Fisher $(p=0.707))$ between the microbiome of active and inactive CD (Figure 2B). Nevertheless, taxonomy-based comparisons of MES-VAT at the phylum, family, and genus level showed significant differences between active and inactive CD. Of note, at the phylum level, we found a significant increase in the relative abundance of Proteobacteria $(52.83 \pm 9.98 \%$ vs. $30.39 \pm 1.75 \%, p=0.0302)$ and a significant decrease in the relative abundance of Bacteroidetes $(17.49 \pm 5.6 \%$ vs. $38.91 \pm 2.5 \%, p=0.0270)$ in active relative to inactive CD (Figure 2C). Additionally, we found a positive correlation between the relative abundance of Proteobacteria and fecal calprotectin $(\mathrm{R}=0.68 ; p=0.0005)$ and $\mathrm{C}$-reactive protein $(\mathrm{R}=0.418 ; p=0.017)$ levels (Figure $2 \mathrm{D})$. 
A.

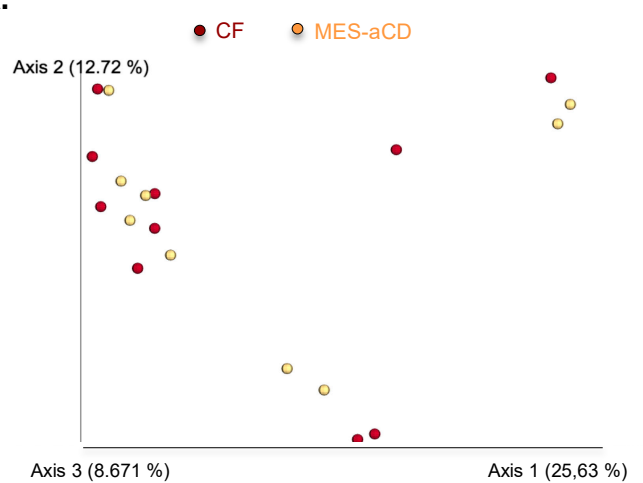

B.

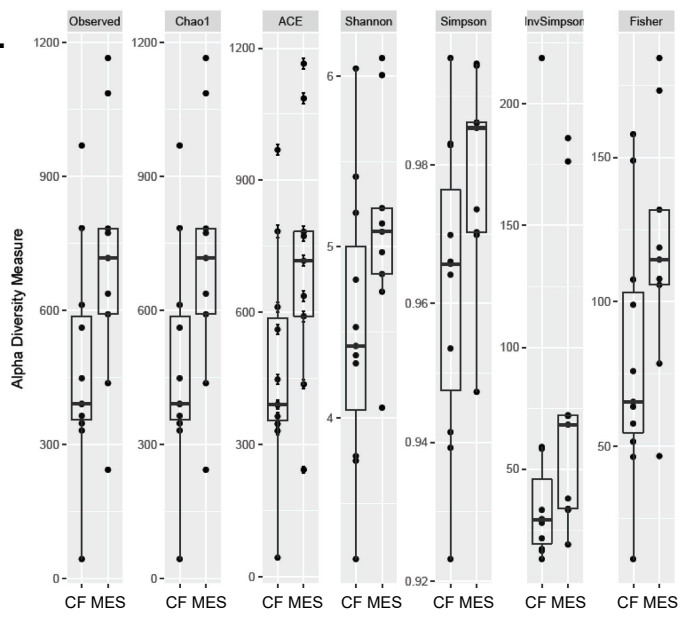

D.

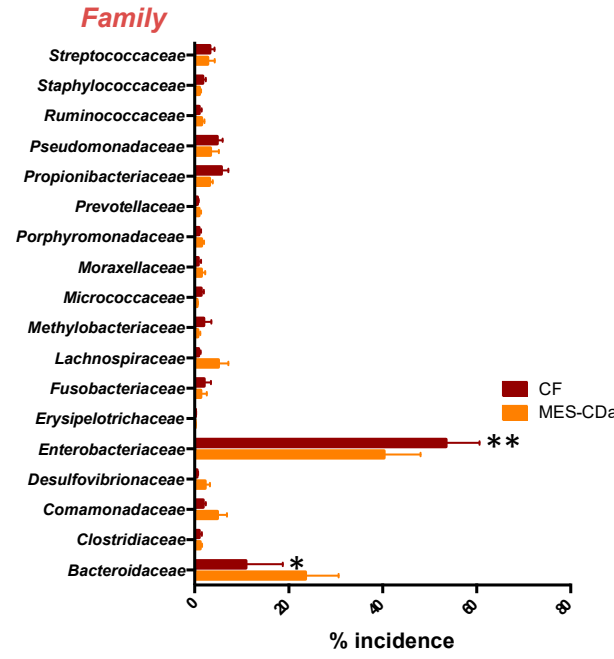

F.

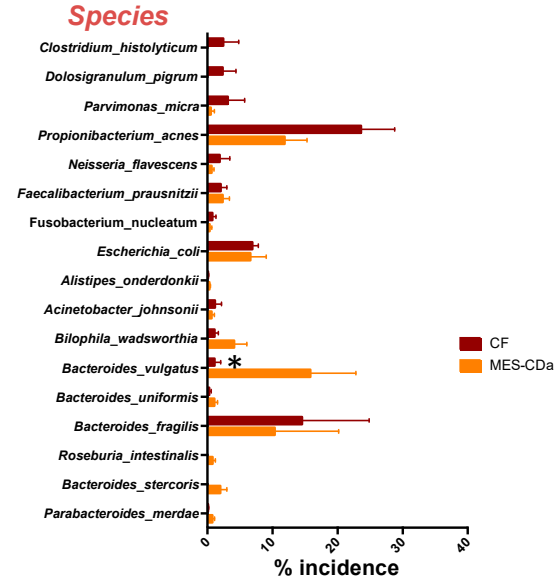

Figure 1. Visceral adipose tissue of patients with active Crohn's disease contains bacterial DNA. (A) Weighted UniFrac principal coordinates analysis of microbiome data from creeping fat-visceral adipose tissue (CF-VAT) and mesenteric (MES)-VAT from eight patients with active Crohn's disease (CD) revealed a similar pattern of clustering. (B) $\alpha$-diversity indices (Observed, Chao1, ACE, Shannon, Simpson, InvSimpson, and Fisher) of the microbiomes of CF-VAT and MES-VAT. (C) Percentage of incidence of phylum, (D) family, (E) genus, and (F) species levels between CF-VAT and MES-VAT of $\mathrm{CD}$ patients. Data information: for $\mathrm{B}$, data are represented in box-and-whisker plot format (whiskers: $\min$ to $\max$ ). For $\mathrm{C}$ to $\mathrm{F}$, values are expressed as mean \pm SEM. Statistical analyses: Two-way analysis of variance with post hoc Bonferroni multiple comparisons test. ${ }^{*}, \mathrm{p}<0.05 ;{ }^{* *}, \mathrm{p}<0.01$. 
A.

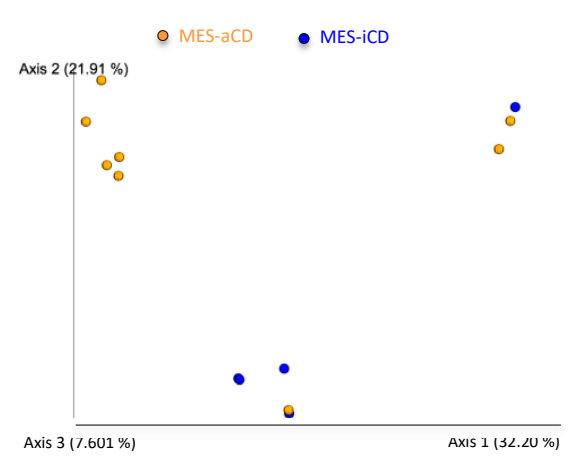

C.

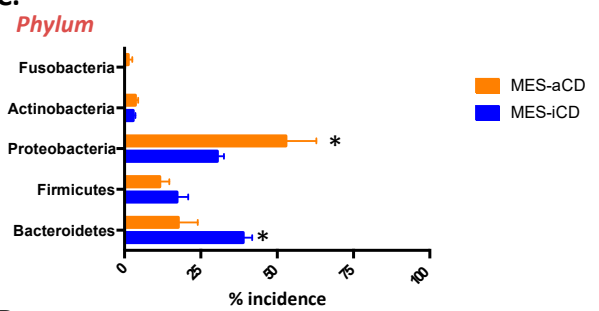

D.

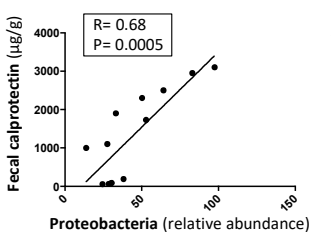

F.

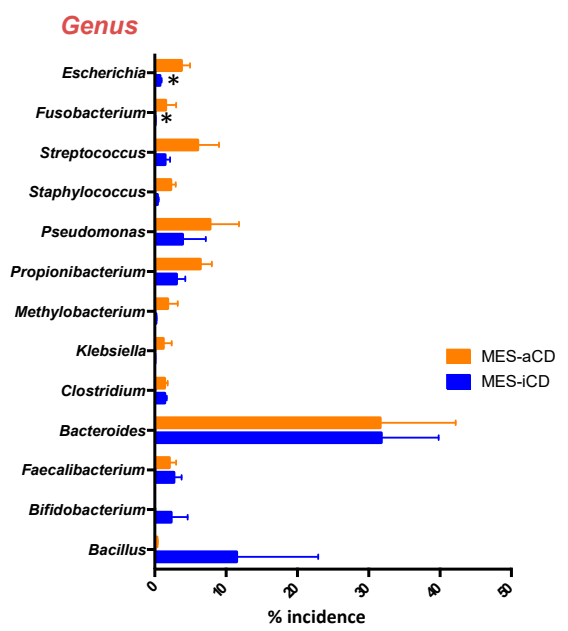

B.

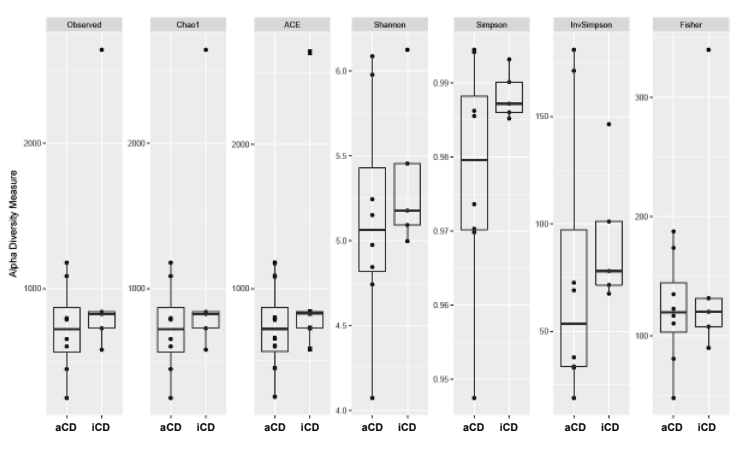

E. Family

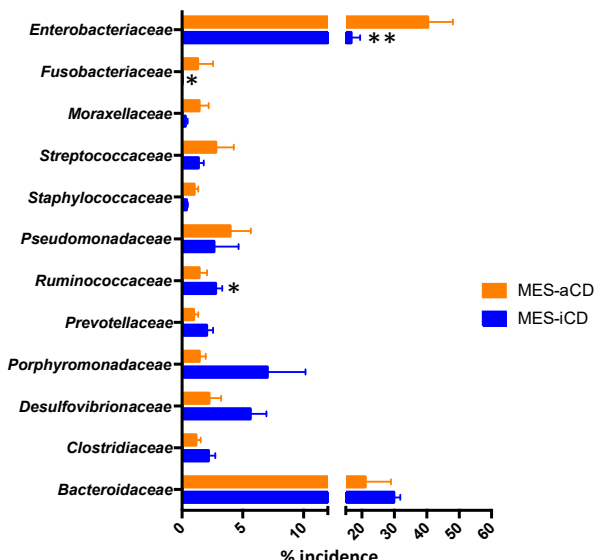

G. Species

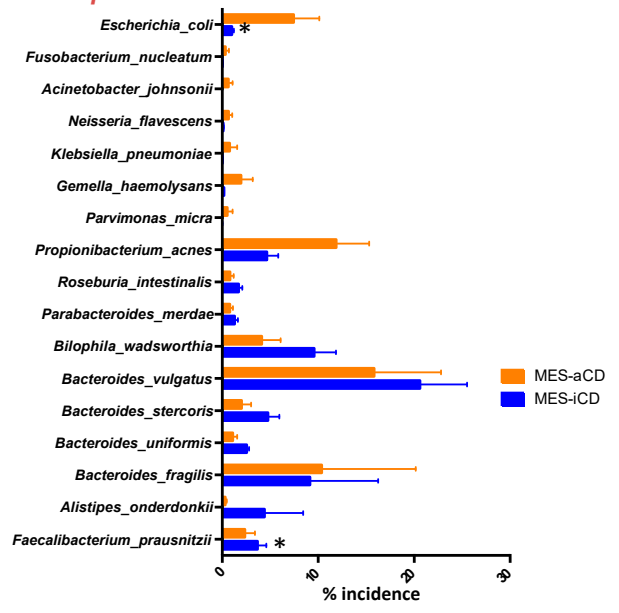

Figure 2. Distinct microbiome signatures in visceral adipose tissue of patients with active/inactive Crohn's disease. (A) Weighted UniFrac principal coordinates analysis of microbiome data from mesenteric-visceral adipose tissue (MES-VAT) of patients with active or inactive Crohn's disease (CD) shows two distinct clusters. (B) $\alpha$-diversity indices (Observed, Chao1, ACE, Shannon, Simpson, InvSimpson, and Fisher) of the microbiome of MES-VAT of patients with active or inactive disease. (C) Percentage of the abundance of phylum levels between MES-VAT of active and inactive patients. (D) Positive correlation between Proteobacteria (relative abundance) and fecal calprotectin or C-reactive protein. Percentage of incidence in (E) family, (F) genus, and (G) species taxon levels in MES-VAT of patients with active or inactive disease. Data information: for $B$, data are represented in box-and-whisker plot format (whiskers: $\min$ to max). For $C$ and $E$ to $G$, values are expressed as mean \pm SEM. Statistical analyses: Two-way analysis of variance with post hoc Bonferroni multiple comparisons test. For D, Pearson's correlation analysis with Bonferroni adjustment was used. ${ }^{*}, p<0.05 ;{ }^{* *}, p<0.001$. 
At the family level, we found that the relative abundance of Enterobacteriaceae $(40.29 \pm 7.73 \%$ vs. $16.74 \pm 2.12 \%, p<0.0001)$ and Fusobacteriaceae $(1.29 \pm 0.73 \%$ vs. $0.03 \pm 0.01 \%, p=0.034)$ was significantly higher in active than in inactive $\mathrm{CD}$, whereas the relative abundance of Ruminococcaceae was significantly lower ( $1.42 \pm 0.64 \%$ vs. $2.78 \pm 0.42 \%, p=0.045)$ (Figure $2 \mathrm{E})$. At the genus level, we found a significant increase in the relative abundance of Escherichia spp. $(4.06 \pm 0.9 \%$ vs. $0.54 \pm 0.02 \%$, $p=0.032)$ and Fusobacterium spp. $(2.4 \pm 0.74 \%$ vs. $0.04 \pm 0.02 \%, p=0.044)$ in active compared with inactive CD (Figure 2F). Lastly, at the species level, we found a significant decrease in the relative abundance of Faecalibacterium prausnitzii $(0.20 \pm 0.16 \%$ vs. $4.48 \pm 0.24 \%, p=0.039)$ and an increase in the relative abundance of Escherichia coli $(7.46 \pm 2.43 \%$ vs. $1.00 \pm 0.18 \%, p=0.0101)$ in the MES-VAT of patients with active $\mathrm{CD}$ compared with their inactive counterparts (Figure $2 \mathrm{G}$ ).

\subsection{Functional Differences in the Gut Microbiota in Mesenteric Adipose Tissue between Active and Inactive Crohn's Disease}

We used the PICRUSt tool to predict the function of the microbial communities based on the $16 \mathrm{~S}$ datasets. No significant differences were detected at the functional level between the CF-VAT and MES-VAT depots in patients with active CD. Remarkably, however, we observed significant functional differences depending on the clinical status of the patient. Metabolism pathway genes (KEGG categories) for energy metabolism $(p=0.003)$ including oxidative phosphorylation $(p=0.011)$ and sulfur metabolism $(p=0.011)$, and also lipid metabolism pathways for fatty acid biosynthesis $(p=0.019)$ and secondary bile acid biosynthesis $(p=0.028)$, were all significantly over-represented in the MES-VAT of patients with active CD compared with their inactive counterparts. Likewise, the MES-VAT of patients with active CD showed significant enrichment in the proportion of genes related to lipopolysaccharide (LPS) biosynthesis $(p=0.006)$, polycyclic aromatic hydrocarbon degradation $(p=0.002)$, and cytochrome P450 ( $p=0.003)$ relative to the MES-VAT of patients with inactive CD (Figure 3). Metagenomic comparison of the study groups showed that gene families linked to pathways in cancer $(p=0.002)$ and colorectal cancer $(p=0.045)$ were significantly increased in the MES-VAT of patients with active $C D$.

Finally, genes related to carbohydrate metabolism $(p=0.011)$ including glycolysis/gluconeogenesis $(p=0.011)$, pentose phosphate pathway $(p=0.030)$, fructose and mannose metabolism $(p=0.045)$, pentose and glucuronate interconversions $(p=0.030)$, linoleic acid metabolism $(p=0.030)$, and propanoate metabolism $(p=0.019)$, as well as genes for alanine, aspartate and glutamate metabolism $(p=0.045)$, nitrogen metabolism $(p=0.045)$ and mineral absorption $(p=0.011)$, were all significantly increased in the MES-VAT of patients with inactive CD relative to their active counterparts (Figure 3). 
A.

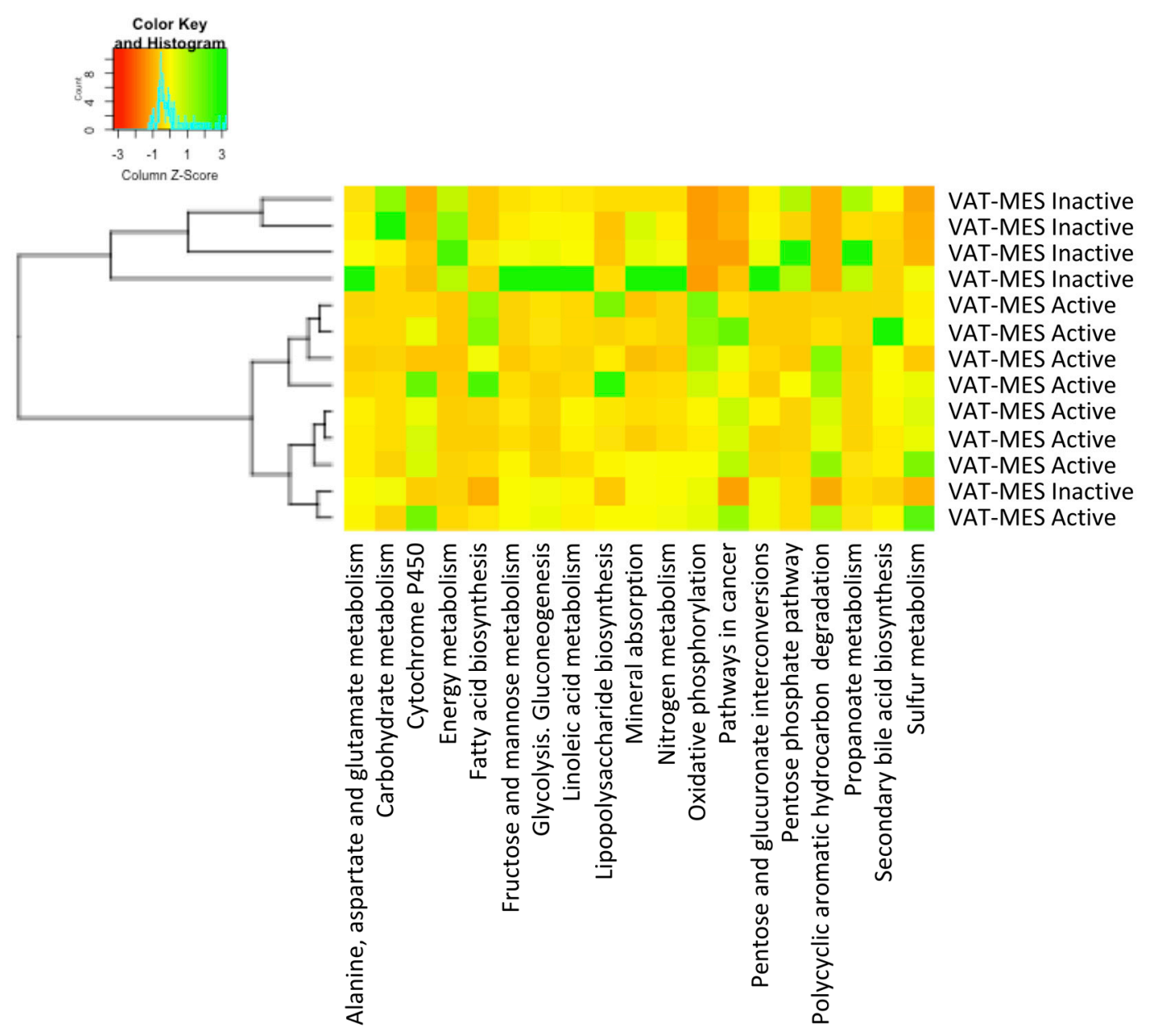

Figure 3. The inferred functional content of gut microbiota in visceral adipose tissue depends on the clinical status of the patients. (A) Heat map of functional content in mesenteric-visceral adipose tissue from patients with active and inactive Crohn's disease was predicted by inferred PICRUSt analysis. $p$-values are corrected for multiple comparisons using the Benjamini-Hochberg method.

\section{Discussion}

In this study we report, for the first time to our knowledge, unique microbiome signatures in the VAT of patients with CD-both in CF-VAT and in MES-VAT. Moreover, our data indicate that clinical remission of $\mathrm{CD}$ does not preclude the presence of particular microbiota composition in mesenteric fat, pointing to this specific tissue as a reservoir for bacteria with the potential to be translocated across a disrupted intestinal barrier.

In recent years, advances in the molecular techniques used to characterize microbial communities have led to a greater appreciation of the diversity of microorganisms inhabiting several unexpected tissues of the human body, including internal tissues. In this line, a distinct diversity of bacteria has been recently described in different AT depots, including breast AT [33], compartments of skin AT [34], and heart AT [35]. Furthermore, the existence of a mesenteric AT microbiota was recently reported in mice using an optimized $16 \mathrm{~S}$ metagenomics sequencing pipeline [36]. An earlier pyrosequencing study in stromal vascular cells from human AT [22] revealed a distinct microbiome in this depot and showed that the diversity of microbiota differed between lean, overweight, and obese persons. By contrast, other authors failed to find a human AT microbiota from obese or normal-weight subjects [23]. This latter observation is in line with the absence of microbial sequences in MES-VAT and SAT from healthy subjects in the present study. Of note, de Goffau and colleagues [24] recently demonstrated 
that the human placenta has no microbiome in a large prospective cohort using both metagenomics and $16 \mathrm{~S}$ amplicon sequencing, which contrasts with what was previously reported [37-39]. The use of aseptic techniques and/or new-generation sequencing methods might explain these contrasting results. Remarkably, a very recent paper by Anhê and colleagues [25] provided evidence for a microbial profile in plasma and liver and three different AT depots (omental, MES-VAT and SAT) of individuals with morbid obesity (mean body mass index $\sim 50 \mathrm{~kg} / \mathrm{cm}^{2}$ ). Interestingly, they found that type 2 diabetes created an extra-intestinal microbial signature, independent of obesity, in the five compartments studied. Intriguingly, the authors observed a greater abundance of Enterobacteriaceae (specifically, Escherichia and Shigella) in the plasma and MES-VAT of subjects with type 2 diabetes. These data are in accord with our findings of Enterobacteriaceae as the most abundant family in the MES-VAT of patients with active $C D$. The aforementioned study thus uncovers a unique organ-specific microbial signature, or potential internal "tissue microbiota", in obesity and type 2 diabetes [40].

$\mathrm{CD}$ and obesity have been proposed as diseases with similar characteristics in terms of manifestations in VAT. Indeed, alterations in body fat distribution and accumulation of intra-abdominal adipose tissue are well-recognized features in $\mathrm{CD}$ and obesity, which are associated with an increased prevalence of systemic inflammation and metabolic disturbances. Moreover, the disruption of the intestinal barrier resulting in a leaky gut has been claimed as a feature common to both diseases. However, whereas the mucosal barrier is only mildly affected in obesity, CD is characterized by severe transmural inflammation with subsequent destruction of the intestinal barrier. Bacteria penetrating the intestinal wall are likely to locate to the mesenteric fat, which could trigger adipocyte hyperplasia and provoke CF-VAT development or persistence [18]. This hypothesis is in line with the findings of our study, i.e., the discovery of bacteria within the $M E S / C F$-VAT of patients with CD.

Of note, we found that the VAT microbiome of patients with CD is a mirror of gut dysbiosis. We detected a broad spectrum of microorganisms in VAT of patients with CD, both in CF-VAT and MES-VAT, with Proteobacteria and Bacteroidetes being the most abundant phyla. It has been described that the intestinal mucosa microbiota in CD differs greatly from that in UC or healthy subjects, with an increase in Bacteroidetes and Proteobacteria [41]. Remarkably, PCoA of the microbiome between $C F$-VAT and MES-VAT of patients with active CD revealed a similar pattern of clustering, indicating no differences in the microbiome between the fat depots. These findings are in agreement with the concept that considers the mesentery as a continuous organ [13,42,43]. Accordingly, CF-VAT and MES-VAT might serve as a bacterial reservoir. By contrast, PCoA of the MES-microbiome between patients with active and inactive disease showed a different cluster pattern, with an increase in Enterobacteriaceae such as Escherichia spp. or Fusobacterium spp. in the MES-VAT of patients with active disease. These microorganisms were previously demonstrated to play a role in the pathogenesis of IBD; specifically, the abundance of fecal Enterobacteriaceae bacteria is augmented both in patients and mice with IBD [44]. E. coli, particularly adherent-invasive E. coli (AIEC) strains, have been isolated from ileal CD biopsies [45] and are also found in patients with UC [46]. Additionally, mucosal samples show more pronounced enrichment than fecal samples [47]. This indicates that the inflammatory milieu in IBD may favor the growth of the Enterobacteriaceae clade. Of note, the anti-inflammatory drug mesalamine was found to attenuate intestinal inflammation and reduce the abundance of Escherichia/Shigella in IBD $[48,49]$.

Fusobacteria is another clade of adherent and invasive bacteria that principally colonize the oral cavity and the gut, and are reported to be present at a higher abundance in the colonic mucosa of IBD patients than in healthy controls [50,51]. When administered by rectal enema in mice, Fusobacterium varium can cause colonic mucosal inflammation [52]. The invasive ability of Fusobacterium bacteria correlates positively with IBD severity in patients [53], indicating that invasive Fusobacterium species may impact IBD pathology. Furthermore, this species has been associated with colorectal cancer, which is the most frequent malignant complication in patients with IBD [54-56]. Fusobacterium spp. produce $\mathrm{H}_{2} \mathrm{~S}$ through cysteine desulfhydrase activity, and we found relevant changes in gene abundance for sulfur metabolism depending on the clinical status of the patients. In agreement with our findings, 
some authors found enrichment for microbial taxa involved in sulfur metabolism, such as Escherichia, Shigella, and Fusobacterium, in fecal samples of patients with active CD [57,58].

There are also specific groups of gut bacteria that might play a protective role against IBD. A range of bacterial species, most notably the genera Lactobacillus, Bifidobacterium, and Faecalibacterium, have been shown to protect the host from mucosal inflammation via several mechanisms, including the stimulation of anti-inflammatory cytokines [59] including IL-10, and the down-regulation of inflammatory cytokines [60]. In this context, our study reveals an increase (although not significant) in the abundance of both Bifidobacterium and Faecalibacterium in patients with inactive relative to active disease. These findings may indicate an increase in beneficial bacteria in patients with remission of the disease. This is consistent with the observations that patients with $C D$ and a low abundance of $F$. prausnitzii in the mucosa are more likely to relapse after surgery [59], whereas restoration of F. prausnitzii after recurrence is associated with maintenance of clinical remission of UC [61]. Of note, we found a significant decrease in F. prausnitzii in the MES-VAT depot of active patients relative to those in remission of the disease. The MES-VAT of patients with active CD also showed a trend for an increase in the abundance of the genus Streptococcus compared with the MES-VAT of patients with inactive CD. Interestingly, the presence of Streptococcus spp. in stool samples before surgery is a predictive marker of future recurrence [6].

In our cohort, analysis of the inferred metagenome showed no significant differences between CF-VAT and MES-VAT of patients with active CD. This finding may indicate that both fat depots, which are contiguous, have a shared tissue microbiota. However, inferred functional analysis of microbiota in VAT from patients with active disease showed enrichment in the abundance of genes involved in LPS biosynthesis relative to patients in remission. LPS is an important component of the outer membrane of Gram-negative bacteria and plays a key role in triggering inflammatory responses in various diseases such as CD. Following our findings, a recent study [62] found that the LPS biosynthesis pathway in mucosal samples of patients with CD was significantly reduced in disease remission, whereas the abundance of pathways involved in glycolysis/gluconeogenesis and starch and sucrose metabolism was increased, suggesting that the induction of remission could partially rectify the gut microbiota dysbiosis and restore metabolic homeostasis.

Our data also showed an increase in genes for nitrogen metabolism; propanoate metabolism; and alanine, aspartate, and glutamate metabolism, in the same line as that found by He and colleagues [62]. Overall, these data indicate that the remission of the disease modifies the microbiota and its metabolic functional patterns in MES-VAT.

Intriguingly, the PICRUSt analysis revealed differences in the abundance of gene families linked to cancer in the samples of active CD patients, which might indicate the genomic potential of the microbes present in MES-VAT of active patients to develop colorectal cancer in these patients. In fact, in this study, we have found in MES-VAT of active patients a significant increase in the abundance of Fusobacterium spp. and Enterobacteriaceae (Escherichia coli), which as previously described may contribute to colorectal carcinogenesis [63-65]. Indeed, several recent studies have shown associations between colorectal cancer and CD activity [66-68] although a direct cause has not yet been demonstrated. The implication of gut microbiota in the link between CD and cancer would be immense, particularly for microbiota-based therapeutics. Nevertheless, we acknowledge the limitation of gene functional profiles inferred from $16 \mathrm{~S}$ sequences, which are predictions only and we interpret these results with caution. Subsequent in-depth studies by whole-genome methods, such as whole community shotgun sequencing and RNA-seq are necessary to increase the insights into the relationships between the functionality of the MES-VAT microbiome and carcinogenesis in active CD patients. Even though MES-VAT from active vs. remission CD patients comes from different intraabdominal localizations, all are representative of visceral fat, and the influence on the results is probably low, however, it must be taken into consideration when interpreting the results. Furthermore, we are aware of the low number of subjects included in this study, and the similar immunosuppressive therapies in both CD groups, 
that may have influenced the microbial signature, nonetheless, changes will be induced in the same sense in both CD groups. All of this warrant further studies in larger cohorts to confirm these results.

In summary, our study points to VAT as a potential barrier against escaped gut bacteria in patients with $C D$, which might drive inflammation in this tissue. We found a microbiome signature enriched in Proteobacteria in the CF-VAT and MES-VAT depots of patients with CD associated with gut dysbiosis. Interestingly, the clinical status of patients altered the abundance of bacteria in VAT, and patients with inactive disease showed a lower abundance of potentially pathogenic bacteria (i.e., Proteobacteria) and a higher abundance of common mucosal bacteria (i.e., Bacteroidetes), as well as a lower abundance of genes involved in LPS biosynthesis compared with patients with active disease. Further studies are needed to determine the pathophysiological role of bacteria (or bacterial DNA) in AT of patients with CD.

\section{Conclusions}

Using 16S-rRNA-based approaches we found for the first time (to our knowledge) that mesenteric adipose tissue is a bacterial reservoir in patients with Crohn's disease. Our data indicate that clinical remission of Crohn's disease does not preclude the presence of a specific microbiota composition in mesenteric fat, pointing to this unique tissue as a well of bacteria with the potential to be translocated across a disrupted intestinal barrier. These novel data revealing a specific microbiome signature in adipose tissue from patients, with a strong relationship with disease activity, might provide the foundations for a new perspective of Crohn's disease that may help to better manage patients.

\section{List of Abbreviations}

$\mathrm{CD}$, Crohn's disease; CF, creeping fat; ASCs, adipose-tissue stem cells; IBD, inflammatory bowel disease; UC, ulcerative colitis; AT, adipose tissue; SAT, subcutaneous adipose tissue; VAT, visceral adipose tissue; MES, mesentery adipose tissue; BMI, body mass index; QUIME II, Quantitative Insights into Microbial Ecology; OTU, operational taxonomic unit; SPSS, Statistical Package for the Social Sciences software; SD, standard deviation; PCoA, Weighted UniFrac principal coordinates analysis; AIEC, adherent-invasive E. coli strains; LPS, lipopolysaccharide.

Supplementary Materials: The following are available online at http://www.mdpi.com/2077-0383/9/8/2448/s1, Figure S1: Creeping fat from $\mathrm{CD}$ patients showed a marked inflammatory infiltration with an increased expression of pro-inflammatory genes.

Author Contributions: C.S., M.Q.-O., L.S.-A., D.M.-F., C.N.-R., E.M.-M. and M.M.R. performed the experiments; M.T.-P. performed biochemical analysis; M.M. (Monica Millan), A.C., B.E., E.E., M.B., M.M. (Margarita Menacho), M.M. (Marc Martí), and F.J.T. carried out part of the study population selection and human sample processing. C.S., M.Q.-O., J.V. and S.F.-V. contributed to the discussion and reviewed the manuscript. C.S., J.V. and S.F.-V. conceived the study, discussed data, and wrote the manuscript. J.V. and S.F.-V. are the guarantors of this work. All authors have read and agreed to the published version of the manuscript.

Funding: This study was supported by grants from the Spanish Ministry of Economy and Competitiveness (PI18/00037, PI15/00143 to C.S.; PI14/00228 and PI17/01503 to J.V., and PI15/00256 to M.Q.-O., RTI2018-093919 to S.F.-V.), co-financed by the European Regional Development Fund (ERDF) and a European Crohn's and Colitis Organization grant to C.S. The Spanish Biomedical Research Center in Diabetes and Associated Metabolic Disorders (CIBERDEM) (CB07708/0012) is an initiative of the Instituto de Salud Carlos III. C.S. acknowledges support from "Ramón y Cajal" program from the Ministerio de Educación y Ciencia (RYC2013-13186), co-financed by the ERDF. S.F.-V. and M.Q.-O. acknowledge support from the "Miguel Servet" tenure track program (CP10/00438; CPII16/0008; and CPI13/00003, respectively) from the Fondo de Investigación Sanitaria, co-financed by the ERDF. M.Q.-O. belongs to the regional "Nicolas Monardes" research program of the Consejería de Salud (C-0030-2018), Junta de Andalucía, Spain, and M.T.-P. is a recipient of a Río Hortega IISCIII fellowship (CM18/00029), both co-financed by the ERDF.

Acknowledgments: We want to particularly acknowledge the patients and the BioBank IISPV (PT17/0015/0029) integrated into the Spanish National Biobanks Network for its collaboration. We thank Dr. Kenneth McCreath for helpful comments on the manuscript.

Conflicts of Interest: The authors declare no conflict of interest. 


\section{References}

1. Carding, S.; Verbeke, K.; Vipond, D.T.; Corfe, B.M.; Owen, L.J. Dysbiosis of the gut microbiota in disease. Microb. Ecol. Health Dis. 2015, 26. [CrossRef] [PubMed]

2. Sartor, R.B.; Mazmanian, S.K. Intestinal microbes in inflammatory bowel diseases. Am. J. Gastroenterol. Suppl. 2012, 1, 15-21. [CrossRef]

3. Mosca, A.; Leclerc, M.; Hugot, J.P. Gut microbiota diversity and human diseases: Should we reintroduce key predators in our ecosystem? Front. Microbiol. 2016, 7, 1-12. [CrossRef] [PubMed]

4. Manichanh, C.; Rigottier-Gois, L.; Bonnaud, E.; Gloux, K.; Pelletier, E.; Frangeul, L.; Nalin, R.; Jarrin, C.; Chardon, P.; Marteau, P.; et al. Reduced diversity of faecal microbiota in Crohn's disease revealed by a metagenomic approach. Gut 2006, 55, 205-211. [CrossRef]

5. Ott, S.J.; Musfeldt, M.; Wenderoth, D.F.; Hampe, J.; Brant, O.; Fölsch, U.R.; Timmis, K.N.; Schreiber, S. Reduction in diversity of the colonic mucosa associated bacterial microflora in patients with active inflammatory bowel disease. Gut 2004, 53, 685-693. [CrossRef]

6. Pascal, V.; Pozuelo, M.; Borruel, N.; Casellas, F.; Campos, D.; Santiago, A.; Martinez, X.; Varela, E.; Sarrabayrouse, G.; Machiels, K.; et al. A microbial signature for Crohn's disease. Gut 2017, 66, 813-822. [CrossRef]

7. Manichanh, C.; Borruel, N.; Casellas, F.; Guarner, F. The gut microbiota in IBD. Nat. Rev. Gastroenterol. Hepatol. 2012, 9, 599-608. [CrossRef]

8. McIlrath, D.C. Diverting ileostomy or colostomy in the management of Crohn's disease of the colon. Arch. Surg. 1971, 103, 308-310. [CrossRef]

9. Zelas, P.; Jagelman, D.G. Loop ileostomy in the management of Crohn's colitis in the debilitated patient. Ann. Surg. 1980, 191, 164-168. [CrossRef]

10. Harper, P.H.; Truelove, S.C.; Lee, E.C.G.; Kettlewell, M.G.; Jewell, D.P. Split ileostomy and ileocolostomy for Crohn's disease of the colon and ulcerative colitis: A 20 year survey. Gut 1983, 24, 106-113. [CrossRef]

11. Li, Y.; Zhu, W.; Gong, J.; Shen, B. The role of the mesentery in Crohn's disease. Lancet Gastroenterol. Hepatol. 2017, 2, 244-245. [CrossRef]

12. Buskens, C.J.; de Groof, E.J.; Bemelman, W.A.; Wildenberg, M.E. The role of the mesentery in Crohn's disease. Lancet Gastroenterol. Hepatol. 2017, 2, 245-246. [CrossRef]

13. Coffey, J.C.; O'Leary, D.P. The mesentery: Structure, function, and role in disease. Lancet Gastroenterol. Hepatol. 2016, 1, 238-247. [CrossRef]

14. Siegmund, B. Mesenteric fat in Crohn's disease: The hot spot of inflammation? Gut 2012, 61, 3-5. [CrossRef] [PubMed]

15. Büning, C.; Von Kraft, C.; Hermsdorf, M.; Gentz, E.; Wirth, E.K.; Valentini, L.; Haas, V. Visceral adipose tissue in patients with Crohn's disease correlates with disease activity, inflammatory markers, and outcome. Inflamm. Bowel Dis. 2015, 21, 2590-2597. [CrossRef]

16. Connelly, T.M.; Juza, R.M.; Sangster, W.; Sehgal, R.; Tappouni, R.F.; Messaris, E. Volumetric fat ratio and not body mass index is predictive of ileocolectomy outcomes in Crohn's disease patients. Dig. Surg. 2014, 31, 219-224. [CrossRef]

17. Gonçalves, P.; Magro, F.; Martel, F. Metabolic inflammation in inflammatory bowel disease: Crosstalk between adipose tissue and bowel. Inflamm. Bowel Dis. 2015, 21, 453-467. [CrossRef]

18. Zulian, A.; Cancello, R.; Ruocco, C.; Gentilini, D.; Di Blasio, A.M.; Danelli, P.; Micheletto, G.; Cesana, E.; Invitti, C. Differences in visceral fat and fat bacterial colonization between ulcerative colitis and Crohn's disease. An in vivo and in vitro study. PLoS ONE 2013, 8, e78495. [CrossRef]

19. Pachón-Peña, G.; Serena, C.; Ejarque, M.; Petriz, J.; Duran, X.; Oliva-Olivera, W.; Simó, R.; Tinahones, F.J.; Fernández-Veledo, S.; Vendrell, J. Obesity determines the immunophenotypic profile and functional characteristics of human mesenchymal stem cells from adipose tissue. Stem Cells Transl. Med. 2016, 5, 464-475. [CrossRef]

20. Serena, C.; Keiran, N.; Ceperuelo-Mallafre, V.; Ejarque, M.; Fradera, R.; Roche, K.; Nuñez-Roa, C.; Vendrell, J.; Fernández-Veledo, S. Obesity and type 2 diabetes alters the immune properties of human adipose derived stem cells. Stem Cells 2016, 34, 2559-2573. [CrossRef] 
21. Serena, C.; Keiran, N.; Madeira, A.; Maymó-Masip, E.; Ejarque, M.; Terrón-Puig, M.; Espin, E.; Martí, M.; Borruel, N.; Guarner, F.; et al. Crohn's disease disturbs the immune properties of human adipose-derived stem cells related to inflammasome activation. Stem Cell Rep. 2017, 9, 1109-1123. [CrossRef] [PubMed]

22. Burcelin, R.; Serino, M.; Chabo, C.; Garidou, L.; Pomié, C.; Courtney, M.; Amar, J.; Bouloumié, A. Metagenome and metabolism: The tissue microbiota hypothesis. Diabetes Obes. Metab. 2013, 15, 61-70. [CrossRef] [PubMed]

23. Zulian, A.; Cancello, R.; Cesana, E.; Rizzi, E.; Consolandi, C.; Severgnini, M.; Panizzo, V.; Di Blasio, A.M.; Micheletto, G.; Invitti, C. Adipose tissue microbiota in humans: An open issue. Int. J. Obes. 2016, 40, 1643-1648. [CrossRef] [PubMed]

24. de Goffau, M.C.; Lager, S.; Sovio, U.; Gaccioli, F.; Cook, E.; Peacock, S.J.; Parkhill, J.; Charnock-Jones, D.S.; Smith, G.C.S. Human placenta has no microbiome but can contain potential pathogens. Nature 2019, 572, 329-334. [CrossRef] [PubMed]

25. Anhê, F.F.; Jensen, B.A.H.; Varin, T.V.; Servant, F.; Van Blerk, S.; Richard, D.; Marceau, S.; Surette, M.; Biertho, L.; Lelouvier, B.; et al. Type 2 diabetes influences bacterial tissue compartmentalisation in human obesity. Nat. Metab. 2020, 2, 233-242. [CrossRef]

26. Gutiérrez, A.; Scharl, M.; Sempere, L.; Holler, E.; Zapater, P.; Almenta, I.; González-Navajas, J.M.; Such, J.; Wiest, R.; Rogler, G.; et al. Genetic susceptibility to increased bacterial translocation influences the response to biological therapy in patients with Crohn's disease. Gut 2014, 63, 272-280. [CrossRef] [PubMed]

27. Laffineur, G.; Lescut, D.; Vincent, P.; Quandalle, P.; Wurtz, A.; Colombel, J.F. Bacterial translocation in Crohn disease. Gastroenterol. Clin. Biol. 1992, 16, 777-781.

28. Best, W.R.; Becktel, J.M.; Singleton, J.W.; Kern, F. Development of a Crohn's disease activity index. Gastroenterology 1976, 70, 439-444. [CrossRef]

29. Van Assche, G.; Dignass, A.; Reinisch, W.; van der Woude, C.J.; Sturm, A.; De Vos, M.; Guslandi, M.; Oldenburg, B.; Dotan, I.; Marteau, P.; et al. The second European evidence-based Consensus on the diagnosis and management of Crohn's disease: Special situations. J. Crohns Colitis 2010, 4, 63-101. [CrossRef]

30. Leiva-Gea, I.; Sánchez-Alcoholado, L.; Martín-Tejedor, B.; Castellano-Castillo, D.; Moreno-Indias, I.; Urda-Cardona, A.; Tinahones, F.J.; Fernández-García, J.C.; Queipo-Ortuño, M.I. Gut microbiota differs in composition and functionality between children with type 1 diabetes and MODY2 and healthy control subjects: A case-control study. Diabetes Care 2018, 41, 2385-2395. [CrossRef]

31. Bhute, S.; Pande, P.; Shetty, S.A.; Shelar, R.; Mane, S.; Kumbhare, S.V.; Gawali, A.; Makhani, H.; Navandar, M.; Dhotre, D.; et al. Molecular characterization and meta-analysis of gut microbial communities illustrate enrichment of prevotella and megasphaera in Indian subjects. Front. Microbiol. 2016, 7, 1-14. [CrossRef] [PubMed]

32. Sanchez-Alcoholado, L.; Castellano-Castillo, D.; Jordán-Martínez, L.; Moreno-Indias, I.; Cardila-Cruz, P.; Elena, D.; Muñoz-Garcia, A.J.; Queipo-Ortuño, M.I.; Jimenez-Navarro, M. Role of gut microbiota on cardio-metabolic parameters and immunity in coronary artery disease patients with and without type-2 diabetes mellitus. Front. Microbiol. 2017, 8. [CrossRef] [PubMed]

33. Hieken, T.J.; Chen, J.; Hoskin, T.L.; Walther-Antonio, M.; Johnson, S.; Ramaker, S.; Xiao, J.; Radisky, D.C.; Knutson, K.L.; Kalari, K.R.; et al. The microbiome of aseptically collected human breast tissue in benign and malignant disease. Sci. Rep. 2016, 6, 1-10. [CrossRef]

34. Nakatsuji, T.; Chiang, H.I.; Jiang, S.B.; Nagarajan, H.; Zengler, K.; Gallo, R.L. The microbiome extends to subepidermal compartments of normal skin. Nat. Commun. 2013, 4. [CrossRef] [PubMed]

35. Pedicino, D.; Severino, A.; Ucci, S.; Bugli, F.; Flego, D.; Giglio, A.F.; Trotta, F.; Ruggio, A.; Lucci, C.; Iaconelli, A.; et al. Epicardial adipose tissue microbial colonization and inflammasome activation in acute coronary syndrome. Int. J. Cardiol. 2017, 236, 95-99. [CrossRef]

36. Lluch, J.; Servant, F.; Païssé, S.; Valle, C.; Valière, S.; Kuchly, C.; Vilchez, G.; Donnadieu, C.; Courtney, M.; Burcelin, R.; et al. The characterization of novel tissue microbiota using an optimized 16S metagenomic sequencing pipeline. PLOS ONE 2015, 10, 1-22. [CrossRef]

37. Aagaard, K.; Ma, J.; Antony, K.M.; Ganu, R.; Petrosino, J.; Versalovic, J. The placenta harbors a unique microbiome. Sci. Transl. Med. 2014, 6. [CrossRef]

38. Antony, K.M.; Ma, J.; Mitchell, K.B.; Racusin, D.A.; Versalovic, J.; Aagaard, K. The preterm placental microbiome varies in association with excess maternal gestational weight gain. Am. J. Obstet. Gynecol. 2015, 212, 653.e1-653.e16. [CrossRef] 
39. Collado, M.C.; Rautava, S.; Aakko, J.; Isolauri, E.; Salminen, S. Human gut colonisation may be initiated in utero by distinct microbial communities in the placenta and amniotic fluid. Sci. Rep. 2016, 6. [CrossRef]

40. Cani, P.D.; Van Hul, M. Microbial signatures in metabolic tissues: A novel paradigm for obesity and diabetes? Nat. Metab. 2020, 2, 211-212. [CrossRef]

41. Gophna, U.; Sommerfeld, K.; Gophna, S.; Doolittle, W.F.; Van Zanten, S.J.O.V. Differences between tissue-associated intestinal microfloras of patients with Crohn's disease and ulcerative colitis. J. Clin. Microbiol. 2006, 44, 4136-4141. [CrossRef] [PubMed]

42. Byrnes, K.G.; Walsh, D.; Walsh, L.; Mirapeix, R.; Lamers, W.; Dockery, P.; McDermott, K.; Coffey, J.C. AB060. 207. Digital reconstruction of human mesentery development. Mesentery Peritoneum 2019, 3, AB060. [CrossRef]

43. Coffey, C.J.; Kiernan, M.G.; Sahebally, S.M.; Jarrar, A.; Burke, J.P.; Kiely, P.A.; Shen, B.; Waldron, D.; Peirce, C.; Moloney, M.; et al. Inclusion of the mesentery in ileocolic resection for Crohn's disease is associated with reduced surgical recurrence. J. Crohns Colitis 2018, 12, 1139-1150. [CrossRef] [PubMed]

44. Lupp, C.; Robertson, M.L.; Wickham, M.E.; Sekirov, I.; Champion, O.L.; Gaynor, E.C.; Finlay, B.B. Host-mediated inflammation disrupts the intestinal microbiota and promotes the overgrowth of enterobacteriaceae. Cell Host Microbe 2007, 2, 119-129. [CrossRef]

45. Darfeuille-Michaud, A.; Boudeau, J.; Bulois, P.; Neut, C.; Glasser, A.-L.; Barnich, N.; Bringer, M.-A.; Swidsinski, A.; Beaugerie, L.; Colombel, J.-F. High prevalence of adherent-invasive Escherichia coli associated with ileal mucosa in Crohn's disease. Gastroenterology 2004, 127, 412-421. [CrossRef]

46. Sokol, H.; Seksik, P.; Rigottier-Gois, L.; Lay, C.; Lepage, P.; Podglajen, I.; Marteau, P.; Doré, J. Specificities of the fecal microbiota in inflammatory bowel disease. Inflamm. Bowel Dis. 2006, 12, 106-111. [CrossRef]

47. Chassaing, B.; Darfeuille-Michaud, A. The commensal microbiota and enteropathogens in the pathogenesis of inflammatory bowel diseases. Gastroenterology 2011, 140, 1720-1728.e3. [CrossRef]

48. Morgan, X.C.; Tickle, T.L.; Sokol, H.; Gevers, D.; Devaney, K.L.; Ward, D.V.; Reyes, J.A.; Shah, S.A.; LeLeiko, N.; Snapper, S.B.; et al. Dysfunction of the intestinal microbiome in inflammatory bowel disease and treatment. Genome Biol. 2012, 13, R79. [CrossRef]

49. Benjamin, J.L.; Hedin, C.R.H.; Koutsoumpas, A.; Ng, S.C.; McCarthy, N.E.; Prescott, N.J.; Pessoa-Lopes, P.; Mathew, C.G.; Sanderson, J.; Hart, A.L.; et al. Smokers with active Crohn's disease have a clinically relevant dysbiosis of the gastrointestinal microbiota. Inflamm. Bowel Dis. 2012, 18, 1092-1100. [CrossRef]

50. Ohkusa, T.; Yoshida, T.; Sato, N.; Watanabe, S.; Tajiri, H.; Okayasu, I. Commensal bacteria can enter colonic epithelial cells and induce proinflammatory cytokine secretion: A possible pathogenic mechanism of ulcerative colitis. J. Med. Microbiol. 2009, 58, 535-545. [CrossRef]

51. Ohkusa, T.; Sato, N.; Ogihara, T.; Morita, K.; Ogawa, M.; Okayasu, I. Fusobacterium varium localized in the colonic mucosa of patients with ulcerative colitis stimulates species-specific antibody. J. Gastroenterol. Hepatol. 2002, 17, 849-853. [CrossRef] [PubMed]

52. Ohkusa, T.; Okayasu, I.; Ogihara, T.; Morita, K.; Ogawa, M.; Sato, N. Induction of experimental ulcerative colitis by Fusobacterium varium isolated from colonic mucosa of patients with ulcerative colitis. Gut 2003, 52, 79-83. [CrossRef] [PubMed]

53. Strauss, J.; Kaplan, G.G.; Beck, P.L.; Rioux, K.; Panaccione, R.; Devinney, R.; Lynch, T.; Allen-Vercoe, E. Invasive potential of gut mucosa-derived fusobacterium nucleatum positively correlates with IBD status of the host. Inflamm. Bowel Dis. 2011, 17, 1971-1978. [CrossRef] [PubMed]

54. Castellarin, M.; Warren, R.L.; Freeman, J.D.; Dreolini, L.; Krzywinski, M.; Strauss, J.; Barnes, R.; Watson, P.; Allen-Vercoe, E.; Moore, R.A.; et al. Fusobacterium nucleatum infection is prevalent in human colorectal carcinoma. Genome Res. 2012, 22, 299-306. [CrossRef] [PubMed]

55. Kostic, A.D.; Gevers, D.; Pedamallu, C.S.; Michaud, M.; Duke, F.; Earl, A.M.; Ojesina, A.I.; Jung, J.; Bass, A.J.; Tabernero, J.; et al. Genomic analysis identifies association of Fusobacterium with colorectal carcinoma. Genome Res. 2012, 22, 292-298. [CrossRef] [PubMed]

56. Yu, G.; Torres, J.; Hu, N.; Medrano-Guzman, R.; Herrera-Goepfert, R.; Humphrys, M.S.; Wang, L.; Wang, C.; Ding, T.; Ravel, J.; et al. Molecular characterization of the human stomach microbiota in Gastric Cancer Patients. Front. Cell. Infect. Microbiol. 2017, 7, 1-11. [CrossRef] [PubMed]

57. Hernandez-Rocha, C.; Kabakchiev, B.; Borowski, K.; Turpin, W.; Boland, K.; Milgrom, R.; Stempak, J.M.; Smith, M.I.; Silverberg, M.S. 257-Higher abundance of bile acid-metabolizing microbiota is associated with type of disease, biopsy location and mucosal inflammation in inflammatory bowel disease patients. Gastroenterology 2019, 156, S-49. [CrossRef] 
58. Carbonero, F.; Benefiel, A.C.; Alizadeh-Ghamsari, A.H.; Gaskins, H.R. Microbial pathways in colonic sulfur metabolism and links with health and disease. Front. Physiol. 2012, 3, 448. [CrossRef]

59. Sokol, H.; Pigneur, B.; Watterlot, L.; Lakhdari, O.; Bermúdez-Humarán, L.G.; Gratadoux, J.-J.; Blugeon, S.; Bridonneau, C.; Furet, J.-P.; Corthier, G.; et al. Faecalibacterium prausnitzii is an anti-inflammatory commensal bacterium identified by gut microbiota analysis of Crohn disease patients. Proc. Natl. Acad. Sci. USA 2008, 105, 16731-16736. [CrossRef]

60. Llopis, M.; Antolin, M.; Carol, M.; Borruel, N.; Casellas, F.; Martinez, C.; Espín-Basany, E.; Guarner, F.; Malagelada, J.R. Lactobacillus casei downregulates commensals' inflammatory signals in Crohn's disease mucosa. Inflamm. Bowel Dis. 2009, 15, 275-283. [CrossRef]

61. Varela, E.; Manichanh, C.; Gallart, M.; Torrejón, A.; Borruel, N.; Casellas, F.; Guarner, F.; Antolin, M. Colonisation by Faecalibacterium prausnitzii and maintenance of clinical remission in patients with ulcerative colitis. Aliment. Pharmacol. Ther. 2013, 38, 151-161. [CrossRef] [PubMed]

62. He, C.; Wang, H.; Liao, W.D.; Peng, C.; Shu, X.; Zhu, X.; Zhu, Z.H. Characteristics of mucosa-associated gut microbiota during treatment in Crohn's disease. World J. Gastroenterol. 2019, 25, 2204-2216. [CrossRef] [PubMed]

63. Iyadorai, T.; Mariappan, V.; Vellasamy, K.M.; Wanyiri, J.W.; Roslani, A.C.; Lee, G.K.; Sears, C.; Vadivelu, J. Prevalence and association of pks+ Escherichia coli with colorectal cancer in patients at the University Malaya Medical Centre, Malaysia. PLoS ONE 2020, 15, e0228217. [CrossRef]

64. Zhou, Z.; Chen, J.; Yao, H.; Hu, H. Fusobacterium and colorectal cancer. Front. Oncol. 2018, 8, 371. [CrossRef] [PubMed]

65. Allen-Vercoe, E.; Jobin, C. Fusobacterium and enterobacteriaceae: Important players for CRC? Immunol. Lett. 2014, 162, 54-61. [CrossRef]

66. Sebastian, S.; Hernández, V.; Myrelid, P.; Kariv, R.; Tsianos, E.; Toruner, M.; Marti-Gallostra, M.; Spinelli, A.; van der Meulen-de Jong, A.E.; Yuksel, E.S.; et al. Colorectal cancer in inflammatory bowel disease: Results of the 3rd ECCO pathogenesis scientific workshop (I). J. Crohns Colitis 2014, 8, 5-18. [CrossRef]

67. dos Santos, S.C.D.; Barbosa, L.A.R. Crohn's disease: Risk factor for colorectal cancer. J. Coloproctol. 2017, 37, 55-62. [CrossRef]

68. Freeman, H.J. Colorectal cancer risk in Crohn's disease. World J. Gastroenterol. 2008, 14, 1810. [CrossRef] 